\title{
Revealing the nature of star forming blue early-type galaxies at low redshift ${ }^{\star}$ (Research Note)
}

\author{
Koshy George $^{1}$ and Kshama Zingade ${ }^{2}$ \\ ${ }^{1}$ Indian Institute of Astrophysics, 2nd Block, Koramangala, 560034 Bangalore, India \\ e-mail: koshy@iiap.res.in \\ 2 Indian Institute of Technology Madras, IIT PO, 600036 Chennai, India \\ e-mail: kshamazings@gmail.com
}

Received 18 August 2014 / Accepted 21 September 2015

\begin{abstract}
Context. Star forming early-type galaxies with blue optical colours at low redshift can be used to test our current understanding of galaxy formation and evolution.

Aims. We want to reveal the fuel and triggering mechanism for star formation in these otherwise passively evolving red and dead stellar systems.

Methods. We undertook an optical and ultraviolet study of 55 star forming blue early-type galaxies, searching for signatures of recent interactions that could be driving the molecular gas into the galaxy and potentially triggering the star formation.

Results. We report here our results on star forming blue early-type galaxies with tidal trails and in close proximity to neighbouring galaxies that are evidence of ongoing or recent interactions between galaxies. There are 12 galaxies with close companions with similar redshifts, among which two galaxies are having ongoing interactions that potentially trigger the star formation. Two galaxies show a jet feature that could be due to the complete tidal disruption of the companion galaxy. The interacting galaxies have high star formation rates and very blue optical colours. Galaxies with no companion could have undergone a minor merger in the recent past. Conclusions. The recent or ongoing interaction with a gas-rich neighbouring galaxy could be responsible for bringing cold gas to an otherwise passively evolving early-type galaxy. The sudden gas supply could trigger the star formation, eventually creating a blue early-type galaxy. The galaxies with ongoing tidal interaction are blue and star forming, thereby implying that blue early-type galaxies can exist even when the companion is on flyby so does not end up in a merger.
\end{abstract}

Key words. galaxies: evolution - galaxies: elliptical and lenticular, cD - galaxies: interactions - galaxies: star formation

\section{Introduction}

Early-type galaxies (ETGs) are morphologically classified as ellipticals or lenticulars based on visual inspection of imaging data. They are observed to be passively evolving stellar systems that follow a tight red sequence when placed on the galaxy colour magnitude diagram (Faber 1973; Visvanathan \& Sandage 1977; Baldry et al. 2004). These galaxies are predominant in dense environments like the centres of galaxy clusters, which are hostile to star formation (Dressler et al. 1987). This observational evidence makes them classic example of systems that formed at very early epochs $(z>2)$, followed by passive evolution (Renzini 2006). However, there is growing evidence from deep surveys in recent years that have demonstrated that the stellar mass in red sequence galaxies has been increasing over the past eight billion years (Bell et al. 2004; Faber et al. 2007; Brown et al. 2007). This could happen because of stellar mass growth through scenarios such as galaxy mergers, tidal interactions, and in situ star formation. These processes operate in the field, increasing the stellar mass of ETGs by accreting more stars and gas, thereby triggering star formation throughout an existing

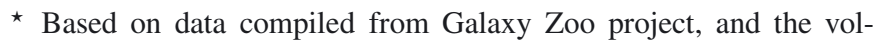
unteers contribution are acknowledged at http://www. galaxyzoo. org/Volunteers.aspx base population of evolved stars. Star formation can then elevate the blue spectral energy distribution of ETGs, which will change their position from the red sequence to the blue cloud on the galaxy colour-magnitude diagram.

Indeed, a sample of such ETGs have been observed to be significantly bluer than the red sequence galaxies (Schawinski et al. 2009, hereafter S09; McIntosh et al. 2014). S09 have reported 204 blue ETGs at low redshifts $(0.02<z<0.05)$, with approximately $L *$ luminosities. The early-type morphology of these galaxies has been confirmed based on visual classifications from the galaxy zoo. They are observed to have a range of velocity dispersions $\left(\sigma<200 \mathrm{~km} \mathrm{~s}^{-1}\right)$, found in low density environments and make up $5.7 \pm 0.4 \%$ of the low-redshift ETG population. Blue ETGs are absent above $u-r$ colour $\sim 2.5$ mag, a region that is populated by red sequence galaxies on the colourmagnitude diagram [S09]. S09 have classified the blue ETGs based on the positions on line diagnostic diagram (Baldwin et al. 1981 ) with $25 \%$ as only star forming, $25 \%$ as both star forming and $\mathrm{AGN}, 12 \%$ as $\mathrm{AGN}$, and $38 \%$ as having no strong emission lines to classify. The star formation rates of the 55 star forming blue ETGs are very high ( 0.45 to $\left.21 M_{\odot} / \mathrm{yr}\right)$ and the colours are too blue for any such systems found in the local universe. We are interested in revealing the mechanisms responsible for star formation in the blue ETGs as it helps in our understanding of galaxy formation and evolution. We speculate a cold gas supply 


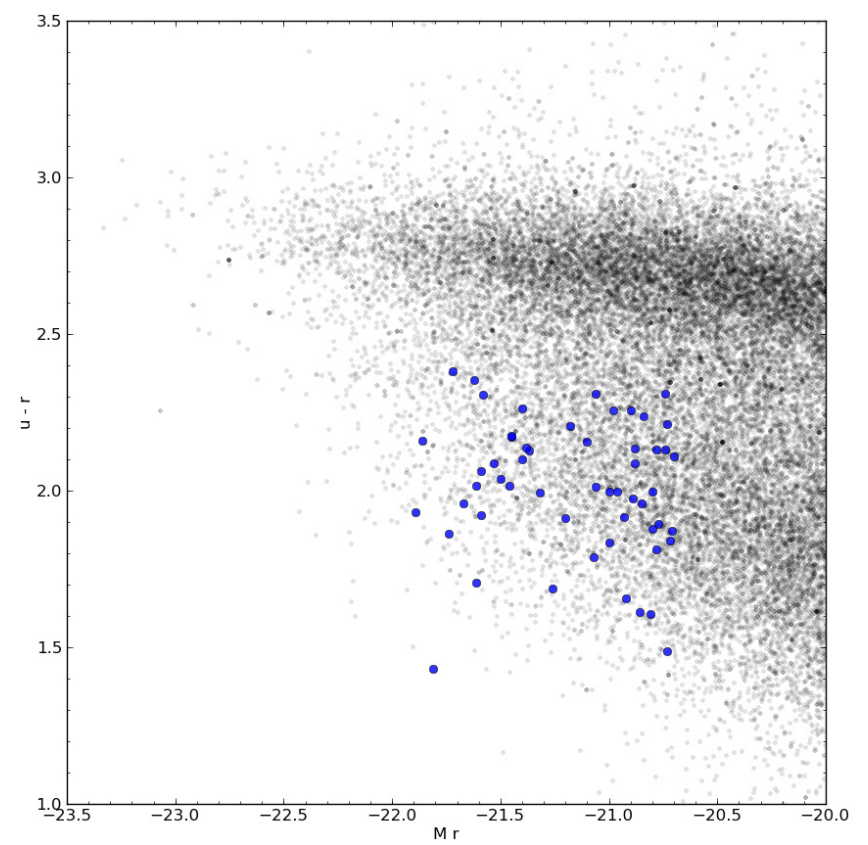

Fig. 1. Colour-magnitude diagram of galaxies in the redshift range of $0.02<z<0.05$. The 55 star forming blue ETGs (blue) are overplotted. Early-type galaxies form a tight red sequence, and the late-type galaxies are scattered in the blue cloud on the colour magnitude diagram.

either from recent gas-rich merger or tidal interaction with a neighbouring gas-rich galaxy, responsible for the observed intense burst of star formation. We report here our initial results based on an optical and ultraviolet imaging analysis of 55 star forming blue ETGs. We adopt a flat Universe cosmology with $H_{\mathrm{o}}=71 \mathrm{~km} \mathrm{~s}^{-1} \mathrm{Mpc}^{-1}, \Omega_{\mathrm{M}}=0.27, \Omega_{\Lambda}=0.73$ (Komatsu et al. 2011).

\section{Data and analysis}

We used SDSS DR7 (Abazajian et al. 2009) $u$ and $r$-band photometric data, $g$-band imaging data, and gri composite images for our analysis. The near ultraviolet galaxy images were taken from the GALEX (Galaxy Evolution Explorer) database (Martin et al. 2005). We constructed the colour-magnitude diagram for galaxies in the redshift range of $0.02<z<0.05$ using the data generated from a volume-limited catalogue of SDSS galaxies with photometric errors less than $0.2 \mathrm{mag}$. The galaxy's absolute magnitudes are based on SDSS petromag, and the colours are derived from SDSS modelMags. Figure 1 shows the colourmagnitude diagram with a blue cloud populated by late-type galaxies and a red-sequence dominated by ETGs. The 55 ETGs studied in this work fall in the blue cloud irrespective of their early-type morphological classification.

\subsection{Optical imaging analysis}

We analysed 55 SDSS gri composite images of $\sim 6^{\prime} \times 6^{\prime}$ field centred on the galaxy to check for any possible close encounters with neighbouring galaxies. The SDSS images have a plate scale of $0.396^{\prime \prime} / \mathrm{pixel}$, and the chosen field corresponds to a physical size of $\sim 144 \mathrm{kpc} \times 144 \mathrm{kpc}$ at $z \sim 0.02$. The region we selected encompasses the virial radius of a typical $L *$ galaxy, a criterion that is crucial for studies of the environments of blue ETGs. The companion galaxies in the $\sim 6^{\prime} \times 6^{\prime}$ field are checked for their proximities, spectroscopic redshifts, and possible interactions with the blue ETG. Companion galaxies within the chosen field that share a minimum redshift increment of $\delta z<0.002\left(\delta v<600 \mathrm{~km} \mathrm{~s}^{-1}\right)$ were classified as neighbours. Based on this criterion, we found a total of twelve blue ETGs with neighbouring galaxies, and this corresponds to $\sim 22 \%$ of the star forming blue ETG sample. The $g$-band images of these galaxies are then analysed for the signs of interactions and the projected distance between the galaxy and the immediate neighbour. The blue ETGs studied here show signatures of recent or ongoing interactions. Two galaxies are found to have ongoing tidal interactions with trails through which stellar material is connected. We could also identify two galaxies with an extended jet feature. Four galaxies are found to have companions with a disturbed morphology that can occur because of the tidal interaction with the blue ETG. The details of twelve blue ETGs with neighbouring companions and of two galaxies having unusual features are given in Table 1 . We adopted the galaxy-naming convention following the S09 objID. Figure 2 shows the images of the representative sample from our analysis.

\subsection{Ultraviolet imaging analysis}

The ultraviolet (UV) spectral energy distribution of a star forming galaxy is dominated by the flux from the young, hot, massive stars. The near UV images of blue ETGs could effectively trace the signatures of ongoing star formation. We checked GALEX near-UV (1750-2750 $\AA$ ) database images of 12 galaxies with neighbours for possible interaction-induced star formation. The GALEX near-UV channel is dominated by the zodiacal light with typical surface brightness levels of $26.5 \mathrm{mag} / \mathrm{arcsec}^{2}$, and the GALEX plate scale (1.5"/pixel) is coarser than that of the SDSS images (Martin et al. 2005). This makes the detection of finer details in near-UV very difficult. There are GALEX data for 10 out of the 12 galaxies with neighbours, and one galaxy (190) with ongoing interaction has no GALEX observation. The contribution from the zodiacal light to the noise level of the images was suppressed by employing statistical smoothing techniques. We performed a Gaussian-smoothing using a kernel of a width of three pixels to enhance the signatures of star formation along the interacting trails. Figure 3 shows the selected nearUV images of two galaxies. Galaxy 119 and the neighbouring galaxy are found to have enhanced emission in near-UV when compared to optical data. This could be due to the sudden burst of intense star formation due to the gravitational interaction between galaxies. We could not detect an interacting trail between the galaxies in the near UV data. However, there can be an exchange of molecular gas (fueling star formation) between the galaxies, which is only detected from radio or sub-mm observations. Galaxy 180, on the other hand, has interaction trails with the companion, and the blobs of enhanced emission are clearly visible in the near-UV images. This could be due to triggered star formation episodes happening within the interacting trails of the galaxies, or we are witnessing the burst of ongoing star formation in the arms of the spiral galaxy that is being stripped by the blue ETG.

\subsection{Genuine case of tidal interaction}

The genuine cases of tidal interactions are seen in Fig. 2. Figure 2a shows Galaxy 119 with a blue companion that appears to be disrupted in morphology owing to the blue ETG. The companion galaxy is having ongoing star formation as is evident from the near-UV image (Fig. 3a). Figure 2b shows Galaxy 180, 
Table 1. Blue ETGs with ongoing tidal interactions and/or observed to have a close companion with equivalent redshifts.

\begin{tabular}{|c|c|c|c|c|c|c|c|c|c|}
\hline $\begin{array}{l}\text { SDSS } \\
\text { ID }\end{array}$ & $\begin{array}{c}\text { S09 } \\
\text { objID }\end{array}$ & $\begin{array}{c}\text { RA } \\
\text { h:m:s }\end{array}$ & $\begin{array}{c}\text { Dec } \\
\mathrm{d}: \mathrm{m}: \mathrm{s}\end{array}$ & $z$ & $\begin{array}{c}M_{r} \\
(\mathrm{mag})\end{array}$ & $\begin{array}{l}u-r \\
(\mathrm{mag})\end{array}$ & $\begin{array}{c}\log \text { SSFR } \\
\left(\mathrm{yr}^{-1}\right)\end{array}$ & $z_{\text {comp }}$ & $\begin{array}{l}\text { Companion } \\
\text { galaxy }\end{array}$ \\
\hline 587725550139408424 & 14 & $12: 35: 02.6$ & $+66: 22: 33.4$ & 0.047 & -21.67 & 1.959 & -10.25 & 0.047 & blue dwarf (strong $\mathrm{H} \alpha$ emission line) \\
\hline 587726100953432183 & 30 & $15: 17: 19.7$ & $+03: 19: 18.9$ & 0.037 & -20.74 & 2.131 & -10.75 & 0.037 & red ETG \\
\hline 587730816286785593 & 61 & $22: 15: 16.2$ & $-09: 15: 47.6$ & 0.038 & -21.61 & 1.707 & -9.64 & - & jet feature \\
\hline 587731500261834946 & 66 & $10: 54: 37.9$ & $+55: 39: 46.0$ & 0.048 & -20.89 & 1.976 & -9.95 & 0.048 & red ETG \\
\hline 587732769982906490 & 92 & $12: 20: 23.1$ & $+08: 51: 37.1$ & 0.049 & -20.88 & 2.086 & -10.23 & 0.049 & blue disturbed disc (strong $\mathrm{H} \alpha$ emission line) \\
\hline 587733412064788620 & 103 & $15: 50: 00.5$ & $+41: 58: 11.2$ & 0.034 & -20.80 & 1.879 & -10.18 & 0.035 & red ETG \\
\hline 587735742615388296 & 119 & $15: 53: 35.6$ & $+32: 18: 20.6$ & 0.050 & -21.07 & 1.789 & -10.07 & 0.050 & blue disturbed spiral galaxy (strong $\mathrm{H} \alpha$ emission line) \\
\hline 587739115234394179 & 139 & 07:56:08.7 & $+17: 22: 50.5$ & 0.030 & -20.73 & 2.211 & -10.62 & 0.028 & blue disturbed galaxy (strong $\mathrm{H} \alpha$ emission line) \\
\hline 587741490904105107 & 160 & $10: 25: 24.7$ & $+27: 25: 06.3$ & 0.050 & -20.98 & 2.256 & -10.42 & 0.049 & red ETG \\
\hline 588007005234856197 & 172 & $08: 17: 56.3$ & $+47: 07: 19.5$ & 0.039 & -21.06 & 2.309 & -10.11 & - & jet feature \\
\hline 588011125186691149 & 180 & $12: 06: 17.0$ & $+63: 38: 19.0$ & 0.040 & -21.26 & 1.686 & -9.59 & 0.040 & blue spiral galaxy interacting (strong $\mathrm{H} \alpha$ emission line) \\
\hline 588017604156784724 & 190 & $14: 14: 33.2$ & $+40: 45: 22.9$ & 0.042 & -20.86 & 1.614 & -9.92 & 0.041 & blue galaxy interacting (strong $\mathrm{H} \alpha$ emission line) \\
\hline 588017991233110227 & 206 & $14: 37: 33.0$ & $+08: 04: 43.0$ & 0.050 & -21.62 & 2.353 & -10.55 & 0.051 & dwarf galaxy \\
\hline 588018254297038899 & 209 & $16: 18: 18.7$ & $+34: 06: 40.1$ & 0.047 & -21.58 & 2.307 & -10.59 & 0.048 & blue disturbed spiral galaxy (strong $\mathrm{H} \alpha$ emission line) \\
\hline
\end{tabular}

Notes. Column (1) is the SDSS DR7 ObjID; Cols. (2) the object ID for the galaxies taken from S09; Cols. (3) and (4) galaxy coordinates (epoch J2000); Col. (5) the spectroscopic redshift of blue ETGs from SDSS; Col. (6) the $r$-band absolute magnitude; Col. (7) the $u-r$ colours; Col. (8) the specific star formation rates; Col. (9) the spectroscopic redshift (zcomp) of nearby companions from SDSS; Col. (10) details obtained from the visual inspection of SDSS gri composite images and the companion-galaxy SDSS spectrum.
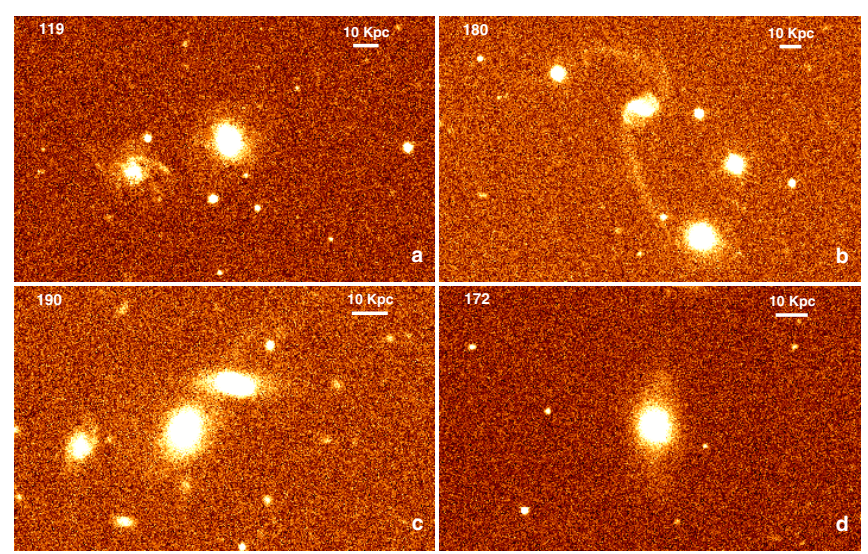

Fig. 2. Blue ETGs SDSS $g$-band (colour-enhanced) images. Representative sample of galaxies with disturbed neighbours a), interacting systems $\mathbf{b})$, c), and galaxies with a jet feature d) are shown. The object ID from S09 is assigned for the blue ETGs in the images. Images are zoomed and scaled to different levels to highlight the interaction features. These images are taken with a $54 \mathrm{~s}$ integration time typical of SDSS imaging data.

which hosts a spiral companion with disrupted morphology. The system is interacting, and the blue ETG have a very high star formation rate of $18 M_{\odot} / y$ r. The spiral companion is being stripped of its spiral arms with tidal trails connecting the galaxies, which is the site of ongoing star formation, as seen in the near-UV image (Fig. 3b). Figure 2c shows Galaxy 190, which is interacting with a blue companion. Figure 2 d shows Galaxy 172 with a jet feature that appears to be a tidally destroyed companion galaxy. We note that the SDSS exposure time ( $54 \mathrm{~s}$ ) is not adequate for bringing out the finer details, which can only be achieved with deep imaging data.

\section{Galaxy colour-magnitude and star formation diagrams}

We plotted 55 blue ETGs in the galaxy optical colour vs. absolute-magnitude diagram and in the absolute-magnitude vs. specific star formation rate diagrams as shown in Fig. 4. The aperture-corrected star formation rate (data from S09) is derived

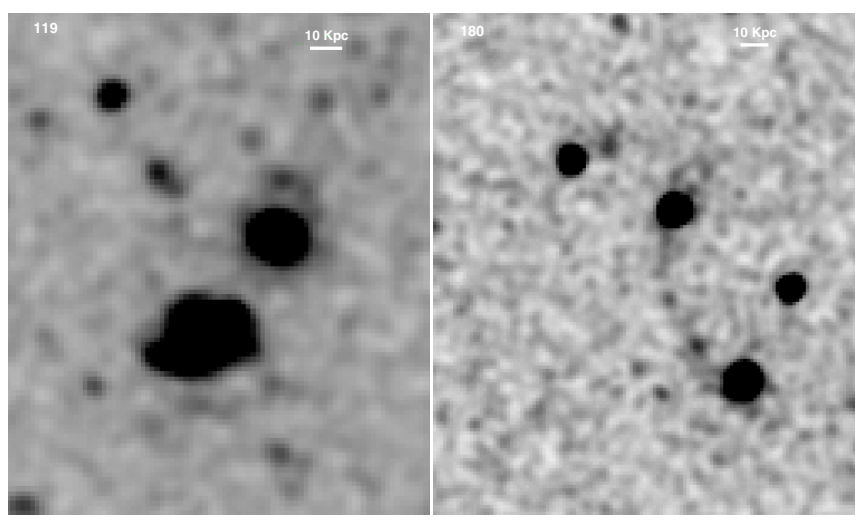

Fig. 3. GALEX near-UV colour-inverted images of selected blue ETGs. a) Galaxy 119 with integration time $\sim 3136 \mathrm{~s}$; b) Galaxy 180 with integration time $390 \mathrm{~s}$.

using the $\mathrm{H} \alpha$ emission line strength measured from the 3 " SDSS fibre spectrum of the inner $3 \mathrm{kpc}$ (at $z \sim 0.05$ ) of the galaxy (see Sect. 3.2 of S09 for details) and scaled for the galaxy-wide star formation. The specific star formation rates were computed using the galaxy stellar mass estimates from the best-fit spectral energy distribution using the flexible stellar population synthesis code (Conroy et al. 2009). The colours were derived from SDSS modelMags, which measures the ratio of the global flux distribution of the galaxy observed in two different filter pass bands. Star formation affects both the $u-r$ colour and the absolute magnitude, making the galaxies appear both brighter and bluer on the colour-magnitude diagram. The galaxies on the magnitude star formation rate diagram show a trend toward brighter galaxies having high star formation rates. It is clear from these diagrams that the interacting galaxies have high star formation rates and very blue colours. This is a strong indication that interaction between galaxies triggers the star formation. (We do caution that it is difficult to make a general conclusion based on data from two interacting systems.) However, it is likely that other systems might have undergone such an interaction in the recent past. This is supported by the distribution of the 12 galaxies with close companions in the diagrams. Galaxies with a jet feature have high star formation rates though the colours are redder for one galaxy than in the rest of the sample. 


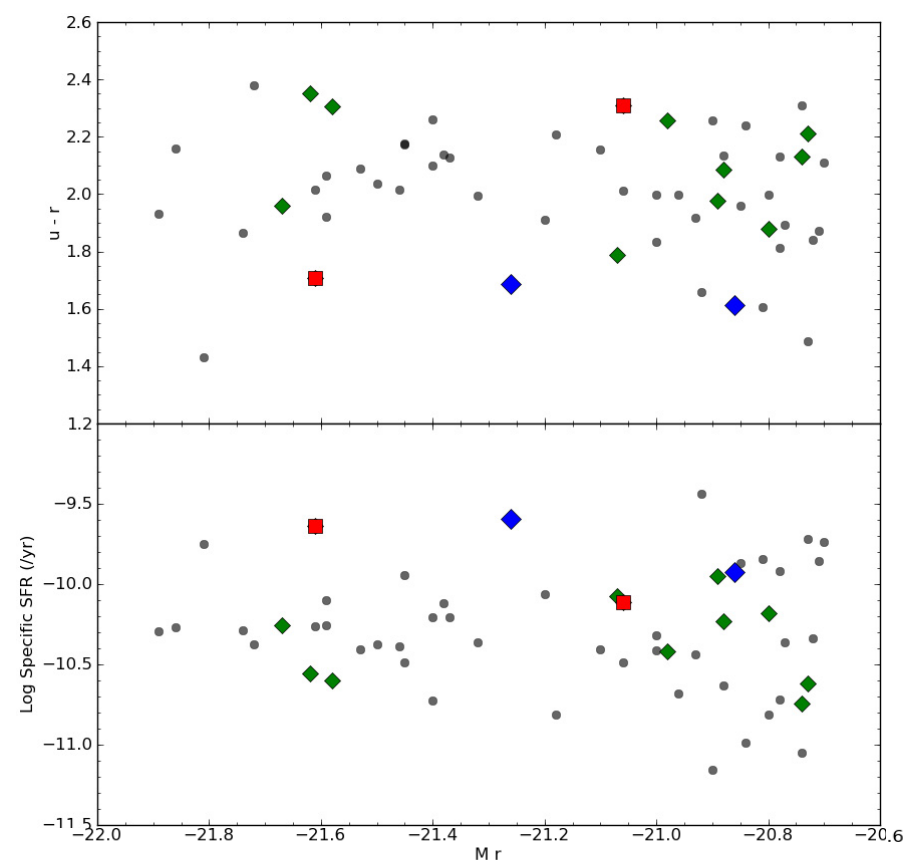

Fig. 4. Galaxy $r$-band absolute magnitude vs. $u-r$ colour and specific star formation rates. The star forming blue ETGs are shown in black, twelve galaxies with neighbours with green diamonds, interacting galaxies with blue diamonds, and the galaxies with jet features with a red square.

\section{Large scale environment analysis}

It has been known since the seminal work of Dressler (1980) that galaxies are prone to morphological transformation in a dense environment. This is supported by the observations of the dominance of ellipticals and S0 galaxies in dense galaxy clusters compared to star forming spiral galaxies. Galaxies get transformed from spirals to ellipticals and S0s when they fall into dense environments. The blue ETGs reported here could undergo preprocessing while in groups or outskirts of galaxy clusters.

The large scale environment of the blue ETGs were analysed to check for an environmental dependence of the observed high star formation rates, whence the blue colours of these galaxies. Galaxies within a region $1 \mathrm{Mpc}$ centred on the cordinates of 55 star forming blue ETGs were searched for the presence of galaxy groups or clusters. The NASA Extragalactic Database (NED) ${ }^{1}$ was used to get the data of neighbouring galaxies. Galaxies were selected above the SDSS limiting magnitude of $g \sim 21$ and within radial velocity of $1200 \mathrm{~km} \mathrm{~s}^{-1}$ around the blue ETG. Figure 5 highlights the results of the analysis as shown in the histogram of galaxies within a $1 \mathrm{Mpc}$ search radius of the blue ETGs studied here. Only galaxies with ten companions and more are shown here. Different bin sizes are chosen to make the histograms on a common velocity scale. Galaxies with high star formation rates are found in varying environmental densities, implying that large scale environmental effects are not seen to enhance star formation in blue ETGs.

\section{Radio data}

The high star formation rates of blue ETGs need an abundant supply of the cold molecular hydrogen that fuels them. In the

\footnotetext{
1 https://ned.ipac.caltech.edu/
}

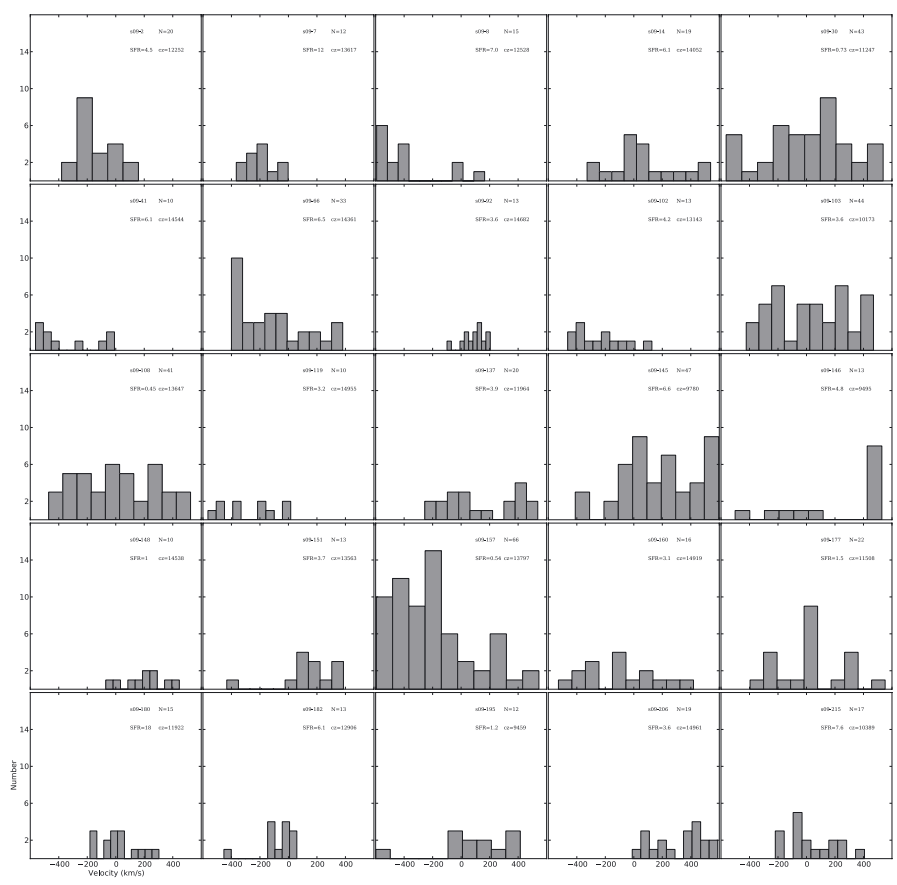

Fig. 5. Histogram of galaxies within a $1 \mathrm{Mpc}$ search radius of blue ETGs. S09 ID, number of galaxies, star formation rate $\left(M_{\odot} / \mathrm{yr}\right)$, and radial velocity $\left(\mathrm{km} \mathrm{s}^{-1}\right)$ are noted on each histogram.

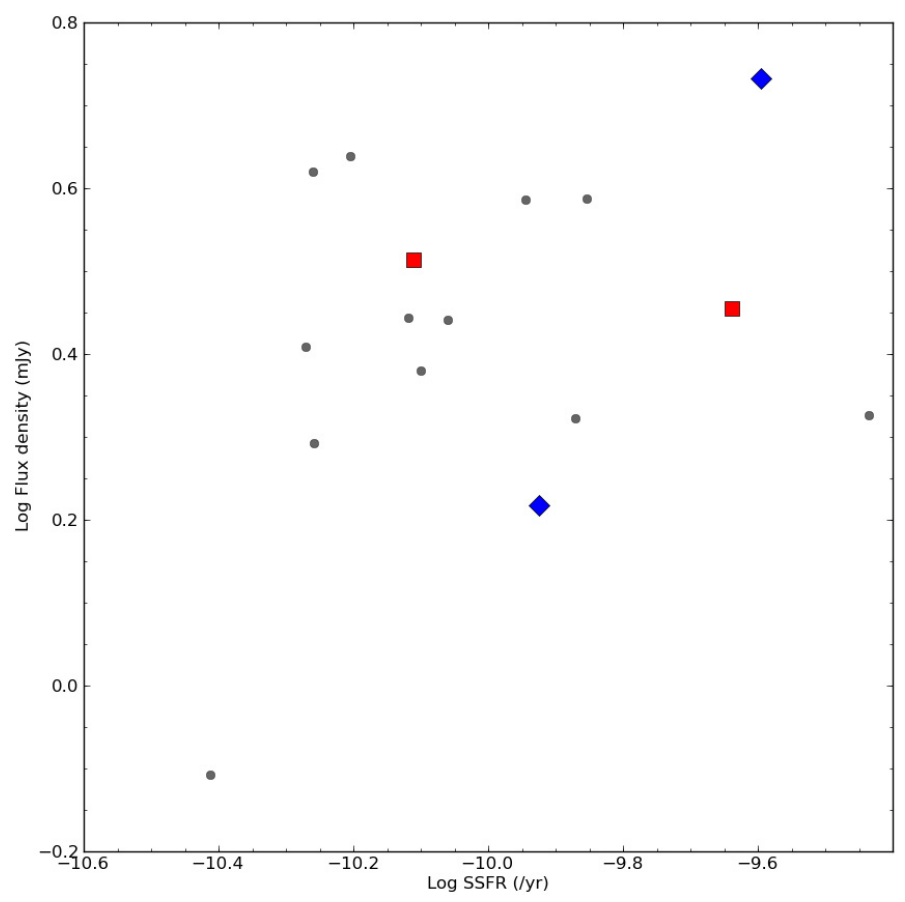

Fig. 6. $1.4 \mathrm{GHz}$ continum flux density of blue ETGs from FIRST survey catalogue plotted against the specific star formation rates. The star forming blue ETGs are shown in black, interacting galaxies in blue diamond, and the galaxies with jet features in the red square.

absence of $\mathrm{HI} / \mathrm{CO}$ data on these galaxies, we checked for other radio data that can be used as a proxy to trace the molecular hydrogen. Data on 16 blue ETGs exist at $1.4 \mathrm{GHz}$ from the FIRST (Faint Images of the Radio Sky at Twenty-cm) survey using Very Large Array (Becker et al. 1995). Radio sources within a separation of 10 arcsec from the galaxy optical coordinates are reported with flux density (in $\mathrm{mJy}$ ) in the $1.4 \mathrm{GHz}$ radio continum. There are 16 galaxies with $1.4 \mathrm{GHz}$ continum flux density above the 
survey detection threshold of $1 \mathrm{mJy}$. Figure 6 shows the plot of specific star formation rates against the detected $1.4 \mathrm{GHz}$ continum flux. It is interesting to note that the galaxies with neighbours do not have flux detection, irrespective of the FIRST survey observations. The radio data can at least trace the star formation (if not the presence of neutral hydrogen) for the 16 galaxies shown here. Future HI observations with VLA/GMRT should shed more light on the neutral hydrogen content and kinematics in blue ETGs.

\section{Discussion}

The existence of morphologically disturbed and peculiar red sequence galaxies are studied in detail by Kaviraj et al. (2011) and Kaviraj (2010) using SDSS and HST imaging data, respectively. These studies conclude that mergers are responsible for the morphological disturbance found in their sample of ETGs. The galaxies studied in our work are blue, and a few have ongoing interactions that make it an ideal case of "caught in the act". That these galaxies are blue and star forming during the phase of interaction (galaxy 180 and 190), point towards a scenario in which such galaxies can exist without a merger scenario. The star formation rates of the blue ETGs are very high, making them brighter and bluer in comparison with the red sequence galaxies. The high star formation rates are only possible with an abundant supply of cold gas. Early-type galaxies are generally gas-poor systems, and they cannot support fuel for such high star formation rates and for the galaxy-scale blue colours found in blue ETGs (Young et al. 2011, 2014). This prompted us to speculate that the fuel for star formation could be accreted from the companion galaxy, which could be a possible scenario at least in the case of galaxies where one can see ongoing interactions. The companion galaxies are significantly bluer in comparison with the blue ETGs. (Details of companion galaxies are given in Table 2.) They could be potentially gas-rich galaxies and show signatures of active star formation (in terms of $\mathrm{H} \alpha$ emission line strength) in the SDSS spectra. The existence of blue ETGs can be explanied by the frosting scenario of residual star formation found in local ETGs on the red sequence, in which an ETG may have triggered star formation over the old base population (Trager et al. 2000; Serra \& Trager 2007). The initial phase of this scenario will rapidly move the galaxy from the red sequence to the blue cloud and will subsequently change the position on the galaxy colour-magnitude diagram. These galaxies will have high star formation rates (depending on the availability of cold gas) and will move back to the red sequence within one billion years once the gas supply or star formation ceases. We speculate that the blue ETGs studied here are at different phases of getting cold gas from the companion and have migrated from the red sequence to the blue cloud. The interacting galaxies could be in the most efficient case of gas accretion, as is evident from the high star formation rate $\left(18 M_{\odot} / y r\right)$. The wide range of star formation rates in these galaxies might correspond to the scenario where we are observing the galaxy at different periods of the star formation activity. The virialisation time scale for a $L *$ galaxy could be smaller than the time scale of ongoing star formation. This, along with the shallow SDSS imaging, explains the reason for not seeing interaction features in many of the star forming blue ETGs.

The galaxies in the local Universe are observed to have gasrich companions with $\mathrm{HI}$ content in the range $\sim 10^{6}-10^{8} \mathrm{M}_{\odot}$ (Sancisi et al. 2008). We consider the case of gas-rich dwarf galaxy Leo $\mathrm{P}$ in the local Universe that have H I content $\sim 10^{6} M_{\odot}$ (Giovanelli et al. 2013). On interaction with a $L * E T G$, this
Table 2. Details of the companion galaxy $r$-band absolute magnitudes and colours and a rough estimate of the projected centre-to-centre distance (in kpc) from the blue ETG.

\begin{tabular}{cccc}
\hline \hline $\begin{array}{c}\text { S09 } \\
\text { objID }\end{array}$ & $\begin{array}{c}\text { Comp } M_{r} \\
(\mathrm{mag})\end{array}$ & $\begin{array}{c}\text { Comp } u-r \\
(\mathrm{mag})\end{array}$ & $\begin{array}{c}\text { Comp } \sim D \\
(\mathrm{kpc})\end{array}$ \\
\hline 14 & -19.64 & 1.23 & 33 \\
30 & -21.90 & 2.93 & 126 \\
66 & -21.05 & 2.63 & 138 \\
92 & -19.21 & 1.10 & 90 \\
103 & -21.53 & 2.77 & 103 \\
119 & -19.03 & 1.00 & 41 \\
139 & -18.41 & 1.41 & 32 \\
160 & -22.15 & 2.89 & 54 \\
180 & -20.35 & 1.30 & 68 \\
190 & -20.07 & 1.26 & 30 \\
206 & -19.03 & 1.90 & 132 \\
209 & -20.80 & 1.83 & 125 \\
\hline
\end{tabular}

galaxy could supply fuel for less than million years with a modest star formation rate of $1 M_{\odot} / y r$ (assuming an efficient case of neutral-to-molecular hydrogen conversion). The fuel will exhaust more rapidly in the case of a star formation rate of the $18 M_{\odot} / y r$ observed in the extreme case of spiral galaxy tidal stripping event reported in this paper (Galaxy 180). This makes a cold gas supply, and the existence of blue ETGs becomes a transient phase in the evolution of a normal ETG and could explain the statistically low fraction [S09] of such galaxies in the local Universe. The extent of the interaction between the companion and the galaxy can be understood from deep optical or/and radio $\mathrm{HI}$ imaging and can be studied for the stellar and gas exchange between the galaxies. The maintenance of the galaxy early-type morphology in a scenario of cold gas accretion from a companion followed by galaxy-scale star formation has to be addressed by detailed simulations. The fate of the interacting companion depends on the mass ratio between the galaxies and can either be a flyby or a major/minor merger. Recent literature points towards minor mergers as responsible for the increase in stellar mass in ETGs residing in low density environments (Kaviraj 2014). The galaxies without a detected companion in SDSS images could have gone through a minor merger phase and can explain the presence of jet features in the $g$-band images, blue colours, and enhanced star formation rates.

\section{Conclusions}

We presented here an analysis of optical and near-UV imaging data of 55 star forming blue ETGs in order to understand the mechanism responsible for star formation. We searched for signatures of recent interactions potentially triggering the star formation. Star forming blue ETGs show tidal trails and close proximity to neighbouring galaxies, which is evidence of ongoing or recent interactions between galaxies. Out of 55 blue ETGs, 12 galaxies have very close blue companions of similar redshifts. Two of these galaxies are ongoing interacting systems with blue colours and high star formation rates. Two galaxies show a jet feature that could be due to tidal disruption of the companion galaxy. Blue ETGs appear to have high star formation rates (as high as $21 M_{\odot} / y r$ ), which is only possible with an abundant cold gas supply from a reservoir. The recent or ongoing interaction with a gas-rich galaxy could be responsible for bringing molecular gas to an otherwise passively evolving red ETG. The gas supply could trigger the star formation, eventually creating a blue ETG. The galaxies with ongoing tidal interaction are 
blue and star forming, which implies that blue ETGs can exist even when the companion is on flyby so does not need to end up in a merger.

Acknowledgements. K.Z. thanks the IIA for a summer intership programme when part of this work was carried out. K.G. is supported by an IndiaTMT postdoctoral fellowship. We thank H.C. Bhatt, Smitha Subramanian, and Blesson Mathew for carefully reading the manuscript and providing critical comments. The SDSS is managed by the Astrophysical Research Consortium for the Participating Institutions. The Participating Institutions are the American Museum of Natural History, Astrophysical Institute Potsdam, University of Basel, University of Cambridge, Case Western Reserve University, University of Chicago, Drexel University, Fermilab, the Institute for Advanced Study, the Japan Participation Group, the Johns Hopkins University, the Joint Institute for Nuclear Astrophysics, the Kavli Institute for Particle Astrophysics and Cosmology, the Korean Scientist Group, the Chinese Academy of Sciences (LAMOST), Los Alamos National Laboratory, the Max Planck Institute for Astronomy (MPIA), the Max Planck Institute for Astrophysics (MPA), New Mexico State University, Ohio State University, University of Pittsburgh, University of Portsmouth, Princeton University, the United States Naval Observatory, and the University of Washington. GALEX is operated for NASA by the California Institute of Technology under NASA contract NAS5-98034.

\section{References}

Abazajian, K. N., Adelman-McCarthy, J. K., Agüeros, M. A., et al. 2009, ApJS, 182,543
Baldry, I. K., Glazebrook, K., Brinkmann, J., et al. 2004, ApJ, 600, 681 Baldwin, J. A., Phillips, M. M., \& Terlevich, R. 1981, PASP, 93, 5 Becker, R. H., White, R. L., \& Helfand, D. J. 1995, ApJ, 450, 559 Bell, E. F., Wolf, C., Meisenheimer, K., et al. 2004, ApJ, 608, 752 Brown, M. J. I., Dey, A., Jannuzi, B. T., et al. 2007, ApJ, 654, 858 Conroy, C., Gunn, J. E., \& White, M. 2009, ApJ, 699, 486

Dressler, A. 1980, ApJ, 236, 351

Dressler, A., Lynden-Bell, D., Burstein, D., et al. 1987, ApJ, 313, 42 Faber, S. M. 1973, ApJ, 179, 731

Faber, S. M., Willmer, C. N. A., Wolf, C., et al., 2007, ApJ, 665, 265 Giovanelli, R., Haynes, M. P., Adams, E. A. K., et al. 2013, AJ, 146, 15 Kaviraj, S. 2010, MNRAS, 408, 170

Kaviraj, S. 2014, MNRAS, 437, L41

Kaviraj, S., Tan, K.-M., Ellis, R. S., \& Silk, J. 2011, MNRAS, 411, 2148

Komatsu, E., Smith, K. M., Dankley, J., et al. 2011, ApJS, 192, 18

Maraston, C., Daddi, E., Renzini, A., et al. 2006, ApJ, 652, 85

Martin, D. C., Fanson, J., Schiminovich, D., et al. 2005, ApJ, 619, L1

McIntosh, D. H., Wagner, C., Cooper, A., et al. 2014, MNRAS, 442, 533

Renzini, A. 2006, ARA\&A, 44, 141

Sancisi, R., Fraternali, F., Oosterloo, T., \& van der Hulst, T. 2008, A\&ARv, 15, 189

Schawinski, K., Lintott, C., Thomas, D., et al. 2009, MNRAS, 396, 818

Serra, P., \& Trager, S. C. 2007, MNRAS, 374, 769

Trager, S. C., Faber, S. M., Worthey, G., \& González, J. J. 2000, AJ, 120, 165

Visvanathan, N., \& Sandage, A. 1977, ApJ, 216, 214

Young, L. M., Bureau, M., Davis, T. A., et al. 2011, MNRAS, 414, 940

Young, L. M., Scott, N., Serra, P., et al. 2014, MNRAS, 444, 3408 